\title{
Expression profile and specific network features of the apoptotic machinery explain relapse of acute myeloid leukemia after chemotherapy
}

Marco Ragusa ${ }^{1}$, Giuseppe Avola², Rosario Angelica ${ }^{1}$, Davide Barbagallo ${ }^{1}$, Maria Rosa Guglielmino ${ }^{1}$, Laura R Duro ${ }^{1}$, Alessandra Majorana', Luisa Statello', Loredana Salito', Carla Consoli², Maria Grazia Camuglia², Cinzia Di Pietro', Giuseppe Milone ${ }^{2}$, Michele Purrello ${ }^{1 *}$

\begin{abstract}
Background: According to the different sensitivity of their bone marrow CD34+ cells to in vitro treatment with Etoposide or Mafosfamide, Acute Myeloid Leukaemia (AML) patients in apparent complete remission (CR) after chemotherapy induction may be classified into three groups: (i) normally responsive; (ii) chemoresistant; (iii) highly chemosensitive. This inversely correlates with in vivo CD34+ mobilization and, interestingly, also with the prognosis of the disease: patients showing a good mobilizing activity are resistant to chemotherapy and subject to significantly higher rates of Minimal Residual Disease (MRD) and relapse than the others. Based on its known role in patients' response to chemotherapy, we hypothesized an involvement of the Apoptotic Machinery (AM) in these phenotypic features.
\end{abstract}

Methods: To investigate the molecular bases of the differential chemosensitivity of bone marrow hematopoietic stem cells (HSC) in CR AML patients, and the relationship between chemosensitivity, mobilizing activity and relapse rates, we analyzed their AM expression profile by performing Real Time RT-PCR of 84 AM genes in CD34+ pools from the two extreme classes of patients (i.e., chemoresistant and highly chemosensitive), and compared them with normal controls.

Results: The AM expression profiles of patients highlighted features that could satisfactorily explain their in vitro chemoresponsive phenotype: specifically, in chemoresistant patients we detected up regulation of antiapoptotic BIRC genes and down regulation of proapoptotic APAF1, FAS, FASL, TNFRSF25. Interestingly, our analysis of the AM network showed that the dysregulated genes in these patients are characterized by high network centrality (i.e., high values of betweenness, closeness, radiality, stress) and high involvement in drug response.

Conclusions: AM genes represent critical nodes for the proper execution of cell death following pharmacological induction in patients. We propose that their dysregulation (either due to inborn or de novo genomic mutations selected by treatment) could cause a relapse in apparent CR AML patients. Based on this, AM profiling before chemotherapy and transplantation could identify patients with a predisposing genotype to MRD and relapse: accordingly, they should undergo a different, specifically tailored, therapeutic regimen and should be carefully checked during the post-treatment period.

\footnotetext{
* Correspondence: purrello@unict.it

'Dipartimento di Scienze BioMediche, Unità di BioMedicina Molecolare

Genomica e dei Sistemi Complessi, Genetica, Biologia Computazionale G

Sichel, Via Santa Sofia 87, 95123 Catania, Italy, EU
} 


\section{Background}

AML is heterogeneous both at the cytogenetic and the molecular level [1,2]. Many of these alterations have prognostic impact on the clinical outcome, especially on resistance to chemotherapy and relapse rates $[1,2]$. As with other tumours, one of the main obstacles to successful chemotherapy is drug resistance [3,4]. Nearly $80 \%$ of AML patients apparently achieve CR following chemotherapy induction, however a high proportion of them relapses and eventually dies of the disease $[5,6]$. To explain MRD or relapses in these patients after chemotherapy, it has been suggested that the bone marrow microenvironment may protect cancer hematopoietic cells, allowing them to resist treatment and survive [7]. This form of resistance (called de novo drug resistance) could be in part attributable to the interaction of the Very Late Antigen (VLA)-4 of leukemic cells with fibronectin within bone marrow stroma [8]. VLA-4 and other adhesion molecules are involved in homing and mobilization of hematopoietic stem cells (HSC) [9]. Treatment strategies, based on blocking adhesion molecules or other proteins involved in homing, might minimize MRD [10]. It has been demonstrated that patients that are good mobilizers are significantly more likely to be responsive to chemotherapy than poor mobilizers [10]. However, unlike other leukemic patients, good mobilizers CR AML patients are subject to significantly higher rates of relapse than patients with lower mobilization capacity; they also have a higher rate of MRD at remission [11-13]. A high ability to mobilize CD34+ cells in peripheral blood (PB) was proved to be an unfavourable prognostic factor, independent of others such as class of cytogenetic risk or FAB morphotype [11-13]. It has been hypothesized that intrinsic or acquired chemoresistance of leukemic bone marrow precursors may be a possible explanation for the association between high mobilization of CD34+ cells and MRD. Furthermore, it has been demonstrated that non-leukemic HSC from AML patients show differential chemosensitivity: this allowed the identification of three categories of patients, characterized by: (i) normal chemosensitivity, (ii) chemoresistance, (iii) increased chemosensitivity, respectively (Milone et al: Chemo-sensitivity of clonigenic marrow precursors in AML patients in complete remission: association with $\mathrm{CD} 34+$ mobilization and with disease-free survival, submitted). A statistically significant correlation between in vitro chemosensitivity of CFU-GM to Etoposide and Mafosfamide, CD34+ cells mobilization and survival was detected: patients with high mobilization capacity showed chemoresistance of their BFU-E and CFU-GM and lower survival rates than patients with normal or low mobilization activity. Dysregulation of the Apoptotic Machinery (AM) plays a primary role in the response to antineoplastic therapy $[14,15]$. In a previous work by some of us, it was demonstrated that the highly interconnected nodes of the AM network (hubs) typically represent the genes with the highest number of genome, transcriptome and proteome alterations in several cancer models [16]. Moreover, we found that most of AM proteins targeted by drugs were characterized by high connectivity; in particular, there was a highly significant association between the betweenness of these proteins and their being targets of drugs [16]. Based on these findings and on our data on AM involvement in anticancer treatment (i.e., Fenretinide in neuroblastoma and Imatinib in CML) [16], we explored the hypothesis that specific AM expression profiles in CR AML patients may determine the differential chemosensitivity, shown in vitro by their bone marrow HSC. The results allow us to propose that there is a relationship between the AM network specific structure and its proneness to dysregulation.

\section{Methods}

The transcriptome of 84 human genes, assigned to the Apoptotic Machinery [17], was analyzed in bone marrow CD34+ cells from a cohort of AML patients in apparent $\mathrm{CR}$ and from normal controls: in all of them, BFU-E, CFU-E, CFU-GM hematopoietic clonogenic precursors were checked for in vitro chemoresistance. Chemosensitivity tests on freshly collected cells and molecular analysis after cell cryopreservation were performed on the same bone marrow samples. AML patients were selected from a cohort of 37 patients studied prospectively, in which the HSC in vitro chemosensitivity was determined. Based on their in vitro drug sensitivity in comparison to normal controls, patients were divided into three groups: (i) normally chemoresponsive, (ii) chemoresistant, (iii) highly chemosensitive. Three patients from the second group and three from the last one were selected for further detailed molecular analysis.

\section{Clinical Features of AML Patients}

Patients' clinical characteristics are reported in Additional File 1. At the time of the study, all patients were in first apparent CR after a cycle of chemotherapy induction and consolidation. Remission was determined by cytogenetic analysis, cytofluorometry, immunophenotyping. The study was approved by the Institutional Ethics Committee and all patients and control donors gave their informed consent for participation in this research.

\section{CD34+ Cells Mobilization in PB of AML Patients}

All patients were treated using a similar chemotherapeutic regimen: one or two induction cycles with 
antracycline, cytarabine (ARA-C) and etoposide, followed by a consolidation cycle with ARA-C and Mitoxantrone in CR patients. A group of normal bone marrow donors $(\mathrm{n}=15)$ was also studied as control. CD34+ mobilization was performed in all CR AML patients after the induction phase. CR was confirmed at that time by immunophenotyping and by cytogenetic assessment in patients showing cytogenetic abnormalities at diagnosis. CD34+ mobilization was carried out during the recovery phase after the first consolidation cycle: G-CSF was administered at the dose of $10 \mathrm{mcg} /$ $\mathrm{kg} /$ day sc, starting 10 days after the end of the consolidation cycle and until the end of the aphaeretic harvest. During mobilization, PB CD34+ cells were daily assessed from the day in which the WBC count in the PB was > $1 \times 10^{9} / \mathrm{L}$; the peak value of the CD34+ cells was used as end point for evaluating the mobilizing ability.

\section{Selection of CD34+ Cells}

Mononuclear cells from bone marrow were purified on a Ficoll gradient at the time of in vitro chemosensitivity assays, frozen in FBS with 10\% DMSO, and stored in liquid nitrogen. After thawing and washing with IMDM (containing 2\% FBS) to remove DMSO, cells were incubated with DNAse to degrade free DNA in solution that would compromise separation efficiency. Separation of CD34+ cells was performed using the CD34 MicroBead Kit (Miltenyi Biotec Macs mini). A final cell purity higher than $80 \%$ was achieved.

\section{In Vitro ChemoSensitivity Assay Of Hematopoietic Precursors}

Bone marrow aspiration in AML patients was carried out between the fourth and sixth week after the end of CD34+ cells mobilization, during disease evaluation. Bone marrow from normal donors was collected through the harvest procedure for allogeneic transplantation. Mononuclear cells were collected from bone marrow samples by Ficoll density gradient separation and divided into six aliquots of 10 million cells in $\mathrm{TC} 199$. After a short incubation at $37^{\circ} \mathrm{C}$, cell aliquots were treated with increasing concentrations of Etoposide (40 and $60 \mathrm{mcg} / \mathrm{ml}$ ) or Mafosfamide (50, 75, 100, 150 $\mathrm{mcg} / \mathrm{ml})$, whereas one aliquot was left untreated as control. Cells were incubated at $37^{\circ} \mathrm{C}$ for $30^{\prime}$, washed in cold TC199 $\left(4^{\circ} \mathrm{C}\right)$, resuspended in IMDM with $2 \% \mathrm{FBS}$, seeded $\left(2 \times 10^{4} / \mathrm{ml}\right)$ in methylcellulose medium supplemented with erythropoietin (HSC-CFU lite with EPO MEDIA MACS Miltenyi Biotec), and incubated in a $37^{\circ} \mathrm{C}, 5 \% \mathrm{CO} 2$, humidified incubator. After $14 \mathrm{~d}$, the colonies (BFU-E, CFU-E, CFU-GM) were analyzed by inverted light microscopy. Drug sensitivity was measured by comparing the number of hematopoietic colonies in samples treated with drugs to untreated controls.

\section{Real Time PCR}

Due to the very low amount of cells at the end of the purification procedure, we had to pool CD34+ cells from three patients with an identical chemophenotype to perform our analysis. Total RNA was extracted by using FastPure ${ }^{\mathrm{Tm}}$ RNA Kit (Takara), according to the Manufacturer instructions. $1 \mu \mathrm{g}$ of total RNA was reverse-transcribed using the High Capacity RNA-tocDNA Kit (Applied Biosystems). 10 ng of cDNA were added to each well of a 96 well PCR array for quantitative PCR (The Human Apoptosis $\mathrm{RT}^{2}$ Profiler $^{\mathrm{TM}}$ PCR Array, SuperArray Bioscience Corporation, MD, USA). The array consisted of 96 primers for 84 protein-encoding $A M$ genes, plus five control genes (ACTB, B2 M, GAPDH, HPRT1, RPL13A), together with PCR and sample quality controls. PCR cycles were performed according to the manufacturer instructions. All experiments were performed in duplicate. Quantitative real time PCR was performed on a Mx3005P ${ }^{\mathrm{rm}}$ QPCR system (Stratagene, La Jolla, CA, USA). The threshold cycle (Ct), defined as the cycle number at which the amount of amplified target reaches a fixed threshold, was obtained for each gene in each sample. The $\mathrm{Ct}$ for each gene in each sample was normalized to the $\mathrm{Ct}$ of control genes, provided in the array, and with respect to each other (normal vs resistant; normal vs sensitive; resistant vs sensitive), according to the $2^{-\Delta \Delta C T}$ method [17]. We also reported our data and the corresponding fold changes applying the following procedure (see Results): if the $2^{-\Delta \Delta C T}$ (RQ) was $\geq 1$, we used the same RQ number (the fold change is positive = up regulation); on the other hand, if RQ was < 1 , we calculated $-1 / R Q$. In this last case, the fold change was negative (down regulation). Analysis and visualization of data was obtained by using the MultiExperiment Viewer 4.4 [18]. To identify differentially expressed genes for the three different comparison reported above, we applied a t-test between subjects $(\Delta \mathrm{Ct})$ by using the following parameters: assumption of equal variance; Alpha (overall threshold $\mathrm{p}$-value) $=0.05$; the $\mathrm{p}$-value was based on t-distribution; the significance was determined by the Adjusted Bonferroni Correction. We reported as up- or down regulated genes having an expression fold change at least $\geq 2$ and $\leq-2$, respectively. Data were partitioned through Hierarchical Clustering, by using the Euclidean distance metric, and the Average Linkage Clustering as linkage method.

\section{Network Analysis}

The global AM network was generated by retrieving, through cytoscape plug-in APID2NET $[19,20]$, all the available experimental interactions among all the $84 \mathrm{AM}$ 
genes analyzed through the Human Apoptosis $\mathrm{RT}^{2}$ Profiler $^{\mathrm{Tm}}$ PCR Array. To create the three specific AM networks, based on three different patient class expression profiles, we applied on APID2NET-interaction maps the three different expression datasets from the chemoresponse classes, using both colour and size gradients of nodes to indicate expression fold changes. Analysis of network centrality was performed by using the plug-in Network Analyzer, which allows to retrieve all the centrality parameters of a node from an established network [21]. The degree of a node inside any biological network mirrors the general topological features of the network, not the real functional importance of the specific node. For this reason, we focused our analysis also on other centrality metrics (i.e., betweenness, closeness, radiality, stress): in biological terms, these may be interpreted as the probability of a protein to be functionally relevant for other proteins and its functional ability to connect different cell nodes. Mathematical details of these centrality metrics are reported by Brandes and Erlebach [22]. Data on functional interactions between genes and drugs were extracted from the Comparative Toxicogenomics Database (CTD), a manually curated repository of specific chemical-gene and chemical-protein interactions in vertebrates and invertebrates from published literature [23]. We inferred the possible correlation between dysregulation of AM genes and their network centrality $(\mathrm{NC})$ or overall drug response (ODR, estimated through the number of literature citations for drugs) by comparing the different NC and ODR in the two different gene expression classes (differentially expressed genes and unchanged genes) by the Wilcoxon Rank Sum test (p-value< 0.05).

\section{Results}

\section{In Vitro ChemoSensitivity Of Hematopoietic Precursors}

In normal subjects, after incubation with maphosphamide at $50 \mathrm{mcg} / \mathrm{mL}$, the mean percentage of normalized residual growth of CFU-GM was $45 \%$ (range $24 \%$ to 57\%). In AML patients, the sensitivity of CFU-GM was highly heterogeneous: by using the values found in normal controls as cut-off points, three groups of patients could be identified. A first group was made of $13 / 37$ (35\%) AML patients, with a sensitivity in the range of normal controls and a mean residual CFU-GM of $33.8 \%$ (normally chemoresponsive patients). In 6/37 patients (16\%), CFU-GM showed increased resistance with a residual CFU-GM above the upper limit of the normal range and a mean of $73.8 \%$ (chemoresistant patients). The third group comprised 18/37 (48\%) AML patients with an increased sensitivity to maphosphamide, a residual growth of CFU-GM below the lower limit of the normal range and a mean of $6.2 \%$ (highly chemosensitive patients).

\section{AM Expression Profile in CD34+ Cells from CR AML Patients}

Quantitative PCR array technology was exploited to examine the transcript levels of 84 AM genes in CD34+ pools from CR AML patients, exhibiting a chemoresistant or a chemosensitive phenotype after in vitro treatment, and control donors. Transcript quantification by the $2^{-\Delta \Delta C T}$ method showed that 23 AM genes from the $\mathrm{Bcl} 2$, Birc, Bnip, caspases, death receptors, death ligands gene families had a nearly similar expression profile in both classes (Tables 1 and 2; Figure 1). On the other hand, 42 AM genes had a different expression profile in patients from either the chemoresistant or the chemosensitive class with respect to controls (Table 1; Figure 1). With the exception of BCL10, BCLAF1, DFFA, that were up regulated, all of these AM genes were down expressed with respect to controls (Table 1; Figure 1). Up regulation of proapoptotic genes BCL10, BCLAF1, DFFA and down regulation of antiapoptotic BAG3, BIRC4, BIRC8, BNIP1, BNIP2, CARD6, CD70, CFLAR, NOL3 in CD34+ bone marrow cells, from both chemoresistant and chemosensitive CR patients, strongly suggest that their AM molecular profile is prone to activation (Tables 1 and 2; Figure 1). We also found that proapoptotic DAPK1, TNFRSF11B, TNFSF8, TP73 genes were down regulated in CD34+ cells from our cohort of CR AML patients (Tables 1 and 2; Figure 1), as already reported for other leukaemia patients after chemotherapy [24-26]; we also observed a down regulation of antiapoptotic BAG3, CD70, NOL3 (Tables 1 and 2 ; Figure 1), that had been previously reported in patients with primary B chronic lymphocytic leukaemia after chemotherapy [27-29]. The comparison of the AM expression profile between resistant and sensitive samples also demonstrated the up regulation of BIRC2 and BIRC3 (two members of the antiapoptotic BIRC family) and down regulation of APAF1, BCL2A1, BCL2L1, CD40LG, CIDEB, FAS, FASL, TNFRSF25, TNFSF10 in the former class (Tables 1 and 3; Figure 1). This expression profile would appropriately explain the in vitro acquired chemoresistant phenotype of CD34+ cells from high mobilizers: in fact, up regulation of BIRC genes was already reported to be related to drug resistance in leukemias and other cancers, as well as the down regulation of proapoptotic APAF1, FAS, FASL, TNFRSF25 [30-34]. Contrary to data previously reported on in vivo resistant AML cells, we detected a down regulation of antiapoptotic BCL2A1 and BCL2L1, respectively, in in vitro chemoresistant CD34+ cells from our cohort of CR patients (Tables 1 and 3; Figure 1).

\section{Specific Features of the AM Network in AML Patients}

The analysis of network centrality showed that AM genes differentially expressed in in vitro resistant CD34+ 
Table 1 Expression fold changes of 84 AM genes in CD34+ cells from CR AML patients

\begin{tabular}{|c|c|c|c|c|c|c|c|}
\hline Genes & $\begin{array}{c}\text { chemoresistant } \\
\text { vs } \\
\text { control }\end{array}$ & $\begin{array}{l}\text { chemosensitive vs } \\
\text { control }\end{array}$ & $\begin{array}{c}\text { chemoresistant } \\
\text { vs } \\
\text { chemosensitive }\end{array}$ & Genes & $\begin{array}{c}\text { chemoresistant } \\
\text { vs } \\
\text { control }\end{array}$ & $\begin{array}{c}\text { chemosensitive } \\
\text { vs } \\
\text { control }\end{array}$ & $\begin{array}{c}\text { chemoresistant } \\
\text { vs } \\
\text { chemosensitive }\end{array}$ \\
\hline ABL1 & $1.05(0.85)$ & $-1.04(1)$ & $-1.11(1)$ & CASP7 & $-2.08(0.14)$ & $-2(0.03)$ & $-1.26(0.17)$ \\
\hline AKT1 & $-1.55(0.15)$ & $-1.05(0.87)$ & $-1.79(0.18)$ & CASP8 & $-15.74(0.006)$ & $-19.29(0.04)$ & $1.01(1)$ \\
\hline APAF1 & $1.35(0.29)$ & $2.66(0.08)$ & $-2.38(0.007)$ & CASP9 & $-1.95(0.20)$ & $-1.46(0.08)$ & $-1.61(0.09)$ \\
\hline BAD & $1.35(0.26)$ & $1.47(0.20)$ & $-1.32(0.46)$ & CD27 & $-1.49(0.24)$ & $-2.33(0.11)$ & $1.29(0.99)$ \\
\hline BAG1 & $1.75(0.34)$ & $1.55(0.47)$ & $-1.07(1)$ & CD40 & $-3.11(0.02)$ & $-4.89(0.01)$ & $1.3(0.50)$ \\
\hline BAG3 & $-41.26(0.0004)$ & $-76.11(0.00009)$ & $1.53(1)$ & CD40LG & $-16.3(0.01)$ & $-2.57(0.04)$ & $-7.67(0.02)$ \\
\hline BAG4 & $-2.34(0.09)$ & $-2.99(0.08)$ & $1.06(1)$ & CD70 & $-10.31(0.002)$ & $-17.03(0.01)$ & $1.37(0.62)$ \\
\hline BAK1 & $-1.11(1)$ & $-1.26(0.47)$ & $-1.06(1)$ & CFLAR & $-51.86(0.002)$ & $-22.01(0.003)$ & $-2.85(0.03)$ \\
\hline BAX & $1.43(0.80)$ & $1.31(0.73)$ & $-1.11(1)$ & CIDEA & $-15.74(0.008)$ & $-19.29(0.009)$ & $1.01(1)$ \\
\hline BCL10 & $3.09(0.007)$ & $3.01(0.007)$ & $1.01(1)$ & CIDEB & $1.26(0.37)$ & $2.55(0.03)$ & $-2.45(0.001)$ \\
\hline BCL2 & $2.15(0.12)$ & $1.9(0.23)$ & $-1.35(0.30)$ & CRADD & $-1.24(0.18)$ & $-1.77(0.07)$ & $1.18(0.52)$ \\
\hline BCL2A1 & $-5.19(0.01)$ & $-2.81(0.02)$ & $-2.23(0.004)$ & DAPK1 & $-15.74(0.0006)$ & $-19.29(0.0002)$ & $1.01(1)$ \\
\hline BCL2L1 & $-3.5(0.007)$ & $1.06(0.44)$ & $-4.47(0.005)$ & DFFA & $3.11(0.00008)$ & $3.01(0.00009)$ & $1.01(1)$ \\
\hline BCL2L10 & $-15.74(0.01)$ & $-19.29(0.02)$ & $1.01(1)$ & FADD & $-5.02(0.004)$ & $-6.41(0.004)$ & $1.06(1)$ \\
\hline BCL2L11 & $1.36(0.25)$ & $1.38(0.26)$ & $-1.22(0.72)$ & FAS & $-7.19(0.01)$ & $-3.53(0.04)$ & $-2.46(0.003)$ \\
\hline BCL2L2 & $-1.25(0.24)$ & $-1.04(1)$ & $-1.44(0.21)$ & FASLG & $-22.58(0.005)$ & $-4.32(0.02)$ & $-6.32(0.01)$ \\
\hline BCLAF1 & $3.12(0.003)$ & $3.04(0.002)$ & $1.01(1)$ & GADD45A & $-2.09(0.16)$ & $-2.69(0.22)$ & $1.06(1)$ \\
\hline BFAR & $3.01(0.0006)$ & $1.84(0.03)$ & $1.01(1)$ & HRK & $-3.75(0.01)$ & $-6.11(0.01)$ & $1.35(0.30)$ \\
\hline BID & $-1.03(0.93)$ & $1.16(0.43)$ & $-1.44(0.21)$ & IGF1R & $-2.29(0.08)$ & $-3.16(0.07)$ & $1.14(1)$ \\
\hline BIK & $1.1(1)$ & $1.47(0.34)$ & $-1.61(0.38)$ & LTA & $-7.34(0.05)$ & $-4.72(0.01)$ & $-1.88(0.12)$ \\
\hline BIRC2 & $3.77(0.006)$ & $-7.26(0.004)$ & $22.63(0.001)$ & LTBR & $-1.38(0.25)$ & $-1.13(0.47)$ & $-1.47(0.27)$ \\
\hline BIRC3 & $-3.02(0.001)$ & $-10.13(0.004)$ & $2.77(0.001)$ & MCL1 & $1.51(0.33)$ & $1.34(0.46)$ & $-1.07(0.51)$ \\
\hline BIRC4 & $-11.77(0.0007)$ & $-15.14(0.0007)$ & $1.06(1)$ & NAIP & $1.96(0.13)$ & $1.39(0.34)$ & $1.16(1)$ \\
\hline BIRC6 & $-1.08(0.85)$ & $1.04(0.73)$ & $-1.37(0.18)$ & NOD1 & $-1.31(0.36)$ & $-1.09(0.94)$ & $-1.44(0.75)$ \\
\hline BIRC8 & $-17.71(0.0005)$ & $-21.71(0.0002)$ & $1.01(1)$ & NOL3 & $-15.74(0.001)$ & $-10.34(0.0003)$ & $-1.84(0.21)$ \\
\hline BNIP1 & $-9.17(0.0008)$ & $-10.41(0.001)$ & $-1.06(1)$ & PYCARD & $1.12(0.48)$ & $1.79(0.07)$ & $-1.93(0.05)$ \\
\hline BNIP2 & $-8.15(0.005)$ & $-7.52(0.007)$ & $-1.31(0.24)$ & RIPK2 & $-1.11(0.21)$ & $-1.69(0.48)$ & $1.26(0.08)$ \\
\hline BNIP3 & $-8.38(0.02)$ & $-9.71(0.03)$ & $-1.04(1)$ & TNF & $2.08(0.18)$ & $2.3(0.31)$ & $-1.34(0.29)$ \\
\hline BNIP3L & $-3.75(0.02)$ & $-4.41(0.01)$ & $-1.03(1)$ & TNFRSF10A & $-6.13(0.013)$ & $-4.03(0.01)$ & $-1.84(0.13)$ \\
\hline BRAF & $-1.09(0.83)$ & $-1.27(0.15)$ & $-1.04(1)$ & TNFRSF10B & $-5.72(0.016)$ & $-4.92(0.02)$ & $-1.4(0.43)$ \\
\hline CARD6 & $-15.74(0.001)$ & $-19.29(0.0008)$ & $1.01(1)$ & TNFRSF11B & $-15.74(0.007)$ & $-19.29(0.007)$ & $1.01(1)$ \\
\hline CARD8 & $-4.55(0.01)$ & $-3.76(0.01)$ & $-1.46(0.19)$ & TNFRSF1A & $2.42(0.13)$ & $1.65(0.11)$ & $1.21(0.76)$ \\
\hline CASP1 & $-1.35(0.45)$ & $1.06(0.48)$ & $-1.74(0.01)$ & TNFRSF21 & $1.1(0.29)$ & $-1.48(0.83)$ & $1.35(0.12)$ \\
\hline CASP10 & $-5.12(0.01)$ & $-6.5(0.012)$ & $1.05(1)$ & TNFRSF25 & $1.07(1)$ & $3.61(0.03)$ & $-4.06(0.03)$ \\
\hline CASP14 & $-25.4(0.03)$ & $-58.89(0.0004)$ & $1.92(0.02)$ & TNFRSF9 & $-15.74(0.03)$ & $-18.38(0.01)$ & $-1.04(1)$ \\
\hline CASP2 & $1.05(1)$ & $1.1(1)$ & $-1.27(1)$ & TNFSF10 & $-1.25(0.12)$ & $1.45(0.08)$ & $-2.2(0.01)$ \\
\hline CASP3 & $1.07(1)$ & $-1.13(0.68)$ & $-1.01(1)$ & TNFSF8 & $-9.04(0.007)$ & $-10.7(0.008)$ & $-1.02(1)$ \\
\hline CASP4 & $-1.47(0.08)$ & $-1.57(0.09)$ & $-1.13(0.72)$ & TP53 & $1.27(0.20)$ & $1.2(0.23)$ & $-1.14(0.70)$ \\
\hline CASP5 & $-12.27(0.002)$ & $-11.71(0.002)$ & $-1.27(0.16)$ & TP53BP2 & $-15.74(0.02)$ & $-19.29(0.003)$ & $1.01(1)$ \\
\hline CASP6 & $1.2(0.34)$ & $1.2(0.43)$ & $-1.21(0.99)$ & TP73 & $-53.32(0.006)$ & $-48.5(0.006)$ & $-1.33(0.70)$ \\
\hline
\end{tabular}

Fold changes of $84 \mathrm{AM}$ gene expression, according to the alternative version of $2^{-\triangle \triangle C T}$ method, for three different comparisons between phenotypic classes: chemoresistant vs control; chemosensitive vs control; chemoresistant vs chemosensitive. When the RQ is $\geq 1$, we reported the same RQ number (positive fold change); when RQ is $<1$, we reported -1/RQ: (negative fold change). We considered as up regulated or down regulated those genes with fold change $\geq 2$ and $\leq$

-2 , respectively. Genes with a fold change between 2 and -2 are considered as unchanged. Adjusted Bonferroni $p$-value is reported between brackets. 
Table 2 Common differentially expressed genes in chemoresistant and chemosensitive classes in comparison with normal controls

\begin{tabular}{|c|c|c|c|}
\hline $\begin{array}{c}\text { Gene } \\
\text { Symbol }\end{array}$ & Category & Expression & NOTES \\
\hline BAG3 & Anti & Down & $\begin{array}{c}\text { Down expression of BAG3 increases apoptosis induced via Bax or Fas by IL-3 deprivation in hematopoietic } \\
\text { cells. Furthermore, BAG3 down modulation is recently shown to enhance the apoptotic response to } \\
\text { chemotherapy with alkylating agents through regulation of CHK2 and CDC2 proteins in human primary } \\
\text { B chronic lymphocytic leukemia cells. }\end{array}$ \\
\hline BCL10 & Pro & Up & $\begin{array}{l}\text { This gene contains a CARD domain, and has been shown to induce apoptosis and to activate NF-kappaB. It is } \\
\text { interesting that deregulation of this gene leads to pathogenesis of hematopoietic malignancy. We detected it } \\
\text { in HSC from our cohort of patients. Its over expression in CR CD34+ cells could be related to their proneness } \\
\text { to death induction. }\end{array}$ \\
\hline BCL2L10 & Pro & Down & $\begin{array}{l}\text { The protein of this gene act as pro-apoptotic regulators that are involved in a wide variety of cellular activities, } \\
\text { interacting with other members of } B C L-2 \text { protein family including } B C L 2, B C L 2 L 1 / B C L-X(L) \text {, and } B A X \text {. }\end{array}$ \\
\hline BCLAF1 & Pro & Up & $\begin{array}{c}\text { This gene encodes a transcriptional repressor that interacts with several members of the BCL2 family. Its } \\
\text { overexpression induces apoptosis. }\end{array}$ \\
\hline $\mathrm{BIRC} 4$ & Anti & Down & $\begin{array}{c}\text { It is a protein which inhibits apoptosis through binding to tumor necrosis factor receptor-associated factors } \\
\text { TRAF1 and TRAF2. It also inhibits at least two members of the caspase family of cell-death proteases, caspase-3 } \\
\text { and caspase-7. }\end{array}$ \\
\hline $\mathrm{BIRC} 8$ & Anti & Down & BIRC8 is involved in the control of apoptosis by direct inhibition of caspase 9 . \\
\hline BNIP1 & Anti & Down & $\begin{array}{l}\text { BNIP genes area members of the BCL2/adenovirus E1B } 19 \mathrm{kd} \text {-interacting protein (BNIP) family. They interact } \\
\text { with the E1B } 19 \mathrm{kDa} \text { protein which is responsible for the protection of virally-induced cell death, as well as, E1B } \\
\qquad 19 \mathrm{kDa} \text {-like sequences of BCL2. }\end{array}$ \\
\hline BNIP2 & Anti & Down & \\
\hline BNIP3 & Pro/Anti & Down & $\begin{array}{c}\text { BNIP3 interacts with the E1B } 19 \mathrm{kDa} \text { protein which is responsible for the protection of virally-induced cell } \\
\text { death, as well as E1B } 19 \mathrm{kDa} \text {-like sequences of BCL2. The dimeric mitochondrial protein is known to induce } \\
\text { apoptosis, even in the presence of BCL2. }\end{array}$ \\
\hline CARD6 & Anti & Down & $\begin{array}{c}\text { This protein is a microtubule-associated protein that has been shown to interact with receptor-interacting } \\
\text { protein kinases and positively modulate signal transduction pathways converging on activation of the inducible } \\
\text { transcription factor NF-kB. }\end{array}$ \\
\hline CASP14 & Pro & Down & $\begin{array}{c}\text { Caspases encode members of the cysteine-aspartic acid protease (caspase) family. Sequential activation of } \\
\text { caspases plays a central role in the execution-phase of cell apoptosis by inducing of either TNF or FAS- } \\
\text { receptor. }\end{array}$ \\
\hline CASP5 & Pro & Down & \\
\hline CD70 & Pro & Down & $\begin{array}{c}\text { This cytokine is also reported to play a role in regulating B-cell activation, cytotoxic function of natural killer } \\
\text { cells, and immunoglobulin synthesis. Its downexpression could confirm that CD34+ cells from these patients } \\
\text { have an expression profile prone to apoptosis and to positively respond to chemotherapy }\end{array}$ \\
\hline CFLAR & Anti & Down & $\begin{array}{c}\text { c-FLIP inhibits caspase } 8 \text { activation and apoptosis mediated by death receptors, such as Fas. Furthermore, } \\
\text { overexpression of c-FLIP potently inhibits apoptosis induced by chemotherapy, suggesting that c-FLIP has a } \\
\text { role in mediating chemoresistance. }\end{array}$ \\
\hline CIDEA & Pro & Down & Cidea that has been shown to activate apoptosis by disrupting a complex consisting of DFF40/CAD. \\
\hline DAPK1 & Pro & Down & $\begin{array}{l}\text { This gene commonly results over expressed in hematopoietic malignancies, but it is down regulated in } \\
\text { patients after chemotherapy. It could be considered a CR marker. }\end{array}$ \\
\hline DFFA & Pro & Up & DFFA is the substrate for caspase- 3 and triggers DNA fragmentation during apoptosis. \\
\hline NOL3 & Anti & Down & $\begin{array}{l}\text { NOL3, an apoptosis suppressor limited to terminally differentiated cells, is induced in human breast cancer and } \\
\text { confers chemo-and radiation-resistance. }\end{array}$ \\
\hline TNFRSF11B & Anti & Down & $\begin{array}{l}\text { Downexpression of this gene in our model could be considered a CR marker: this gene commonly results over } \\
\text { expressed in hematopoietic malignancies, but it is down regulated in patients after chemotherapy }\end{array}$ \\
\hline TNFRSF9 & Anti & Down & $\begin{array}{l}\text { This receptor contributes to the clonal expansion, survival, and development of T cells. It can also induce } \\
\text { proliferation in peripheral monocytes, enhance T cell apoptosis induced by TCR/CD3 triggered activation. }\end{array}$ \\
\hline TNFSF8 & Anti & Down & $\begin{array}{c}\text { This gene commonly results over expressed in hematopoietic malignancies, but it is down regulated in } \\
\text { patients after chemotherapy. It could be considered a CR marker. }\end{array}$ \\
\hline TP53BP2 & Pro & Down & $\begin{array}{l}\text { This protein is localized to the perinuclear region of the cytoplasm, and regulates apoptosis and cell growth } \\
\text { through interactions with other regulatory molecules including members of the p53 family. }\end{array}$ \\
\hline TP73 & Pro & Down & $\begin{array}{c}\text { This gene commonly results over expressed in hematopoietic malignancies, but it is down regulated in } \\
\text { patients after chemotherapy. It could be considered a CR marker. }\end{array}$ \\
\hline
\end{tabular}

The biological functions, AM category (pro-or anti apoptotic activity), and our comments, based on our findings, are reported for each gene 
cells were positively associated to higher network centrality respect to unchanged genes (Wilcoxon Rank Sum test): betweenness $(\mathrm{p}$-value $=0.043)$, closeness $(\mathrm{p}$-value $=$ $0.019)$, radiality $(\mathrm{p}$-value $=0.019)$, stress $(\mathrm{p}$-value $=0.041)$ (Figure 2). Moreover, these genes were more tightly associated to drug response than unchanged genes ( $\mathrm{p}$-value $=$ 0.003) (Figure 2). These network centrality parameters are biologically more important than the simple network degree of a node, because they demonstrate the ability of a protein to functionally connect to and be relevant for several others within a complex signalling network [35]. By comparing chemoresistant and chemosensitive classes, we found that dysregulated genes are frequently critical nodes of the AM network and drug-related genes. Moreover, by integrating the expression values of the three profiles into the network structure, we found that the expression modification of some nodes could lead to important alterations of the network topology (Figure 3). When compared to those from normal donors, the networks of resistant and sensitive classes appeared quite similar; on the contrary, the direct comparison between resistant and sensitive classes identified differences that could unbalance the functional equilibrium of the AM network (e.g., the down regulation of many proapoptotic genes) (Table 3, Figure 3). These altered network structures could explain the different AM behaviour as well as the different in vitro drug sensitivity and the clinical phenotype of patients (i.e., MRD and relapse).

\section{Discussion}

Drug resistance is a major cause of failure in cancer treatment $[7,36]$. Chemotherapy exerts on tumour cells a strong selective pressure: accordingly, their survival relies on a dynamic mutation process, possibly leading to an ever fitting chemoresistance phenotype [37]. The bone marrow microenvironment is a secluded and potentially safe body niche, in which cancer cells can be protected against chemotoxic agents. Mobilization from this compartment is a complex process, that requires the orchestrated participation of several molecules as chemokines, adhesion molecules, and their downstream targets $[10,38]$. The molecular events that regulate HSCs' engraftment and mobilization are still incompletely defined. The precise mechanism of mobilization by the Colony Stimulating Factors (CSF), the factor most commonly used to mobilize hematopoietic cells from bone marrow to $\mathrm{PB}$, remains unsatisfactorily characterized $[39,40]$. CSF binding to its receptor leads to the activation of several downstream signalling cascades affecting cell survival, proliferation, differentiation, migration [41]; moreover, it is well known that CSF suppresses apoptosis in both normal HSCs and cancer cells [42]. In our experiments, we observed that CR patients with high CD34+ mobilization activity showed in vitro 
Table 3 Differentially expressed genes in chemoresistant patients respect to chemosensitive patients

\begin{tabular}{|c|c|c|c|}
\hline $\begin{array}{c}\text { Gene } \\
\text { Symbol }\end{array}$ & Category & Expression & NOTES \\
\hline APAF1 & pro & Down & It is a component of apoptosome. The apoptosome has a role in chemioresistance in pancreatic cancer. \\
\hline $\mathrm{BCL} 2 \mathrm{~A} 1$ & anti & Down & $\begin{array}{l}\text { This gene is able to reduce the release of pro-apoptotic cytochrome c from mitochondria and block caspase } \\
\text { activation. This gene is a direct transcription target of NF-kappa B and it acts in response to cytokine TNF } \\
\text { and IL-1. }\end{array}$ \\
\hline $\mathrm{BCL} 2 \mathrm{~L} 1$ & pro/anti & Down & $\begin{array}{l}\text { It acts as anti- or pro-apoptotic regulators. This gene is located on mitochondrial membrane and control VDAC } \\
\text { opening, regulating the release of cytochrome } C \text { by mitochondria. }\end{array}$ \\
\hline $\mathrm{BIRC2}$ & anti & Up & $\begin{array}{l}\text { This gene is a member of a family of proteins that inhibits apoptosis by binding to tumor necrosis factor } \\
\text { receptor-associated factors TRAF1 and TRAF2. }\end{array}$ \\
\hline $\mathrm{BIRC3}$ & anti & Up & $\begin{array}{l}\text { This gene is a member of a family of proteins that inhibits apoptosis by binding to tumor necrosis factor } \\
\text { receptor-associated factors TRAF1 and TRAF2. In cells resistant to alkylating agent, it blocks through regulation } \\
\text { of CHK2 and CDC2 proteins. }\end{array}$ \\
\hline CD40LG & pro & Down & It regulates B cell function by engaging CD40 on the B cell surface. \\
\hline CIDEB & pro & Down & $\begin{array}{l}\text { CIDEB triggers DNA fragmentation and nuclear condensation. Its expression could be associated to resistance } \\
\text { to apoptosis. }\end{array}$ \\
\hline FAS & pro & Down & $\begin{array}{l}\text { FAS/FASL ratio, after receiving chemotherapy, indicates chemosensitivity in several tumoral models. In addition, } \\
\text { the decreasing ratio during chemotherapy treatment, despite the initial values, is related to acquired } \\
\text { chemoresistance. }\end{array}$ \\
\hline FASLG & pro & Down & \\
\hline TNFRSF25 & pro & Down & $\begin{array}{l}\text { This receptor has been shown to stimulate NF-kappa B activity and regulate cell apoptosis. The signal } \\
\text { transduction of this receptor is mediated by various death domains contained by adaptor proteins. }\end{array}$ \\
\hline TNFSF10 & pro & Down & $\begin{array}{l}\text { This protein preferentially induces apoptosis in transformed and tumoral cells and it is expressed at a } \\
\text { significant level in the most of normal tissues. Its down regulation could impair the apoptosis induction. }\end{array}$ \\
\hline
\end{tabular}

The biological functions, AM category (pro- or anti apoptotic activity), and our comments, based on our findings, are reported for each gene.

chemoresistance of their BFU-E and CFU-GM. This was coupled to a poorer prognosis, due to an increased relapse rate respect to normally or highly chemosensitive patients. Intriguingly, chemoresistant patients showed an AM expression profile that strongly suggested the involvement of the AM network in their in vitro drug resistance. Thus, a relationship between mobilization from bone marrow, apoptosis induction and chemoresistance seems to exist in these patients. The chemoresistant class is characterized by up regulation of Birc genes (BIRC2 and BIRC3), that inhibit the action of caspases, and down expression of proapoptotic genes as APAF1 (an apoptosome component), FAS, FASL, TNFRSF25, TNFSF10. This molecular phenotype could lead to a failure in activating apoptosis in response to chemotherapy [30-34]. It is well known that GM-CSF inhibits Fas-induced apoptosis and stimulates expression of BIRC family members $[43,44]$. This protective effect of CSF can also explain the lower complete remission rates after chemotherapy in AML patients, whose cells have a higher responsiveness to hematopoietic viability factors in vitro [45]. Our data demonstrate a down regulation of proapoptotic genes CD40LG, CIDEB, TNFSF10 in chemoresistant AML patients, previously not reported in association with drug resistance. Furthermore, differently from previous reports that in vivo resistant AML cells express high levels of BCL2A1 and BCL2L1 [30,34], we found that both genes are down regulated in chemoresistant AML patients in comparison to those from the chemosensitive class. We suggest that this discrepancy is due to the specific features of the different cell types analyzed: we analyzed in vitro selected chemoresistant, possibly non-leukemic CD34+ cells from CR AML patients, whereas Eisele et al. and Valdez et al. studied myeloid blasts from AML patients [46] or mononuclear cells from AML patients [31], respectively. Why are some CR AML patients good responders to CSF and others are not? The continued pressure of chemotherapy may induce or select genomic mutations, able to alter the cytokine cross-talking network between cell migration and apoptosis: this would result in an acquired drug resistance phenotype [8]. We may otherwise assume that within the HSC population of some CR patients, few leukemic cells survive chemotherapy and persist under the CR threshold to be eventually selected by the mobilizing treatment $[47,48]$. The presence of a substantial number of MRD cells could influence the microenvironment and enhance their protective effect from chemotherapy damage. This would explain not only the high relapse rate, but also the high mobilization activity through a reduced myelotoxicity induced by chemotherapy. Based on our results, we suggest that the presence of MRD can lead to a modulation of BM microenvironment that could cause dysregulation of some components of the AM network in HSC. Interestingly, by comparing both the resistant and the sensitive class with control samples, we noticed that 23 AM genes showed an expression profile 


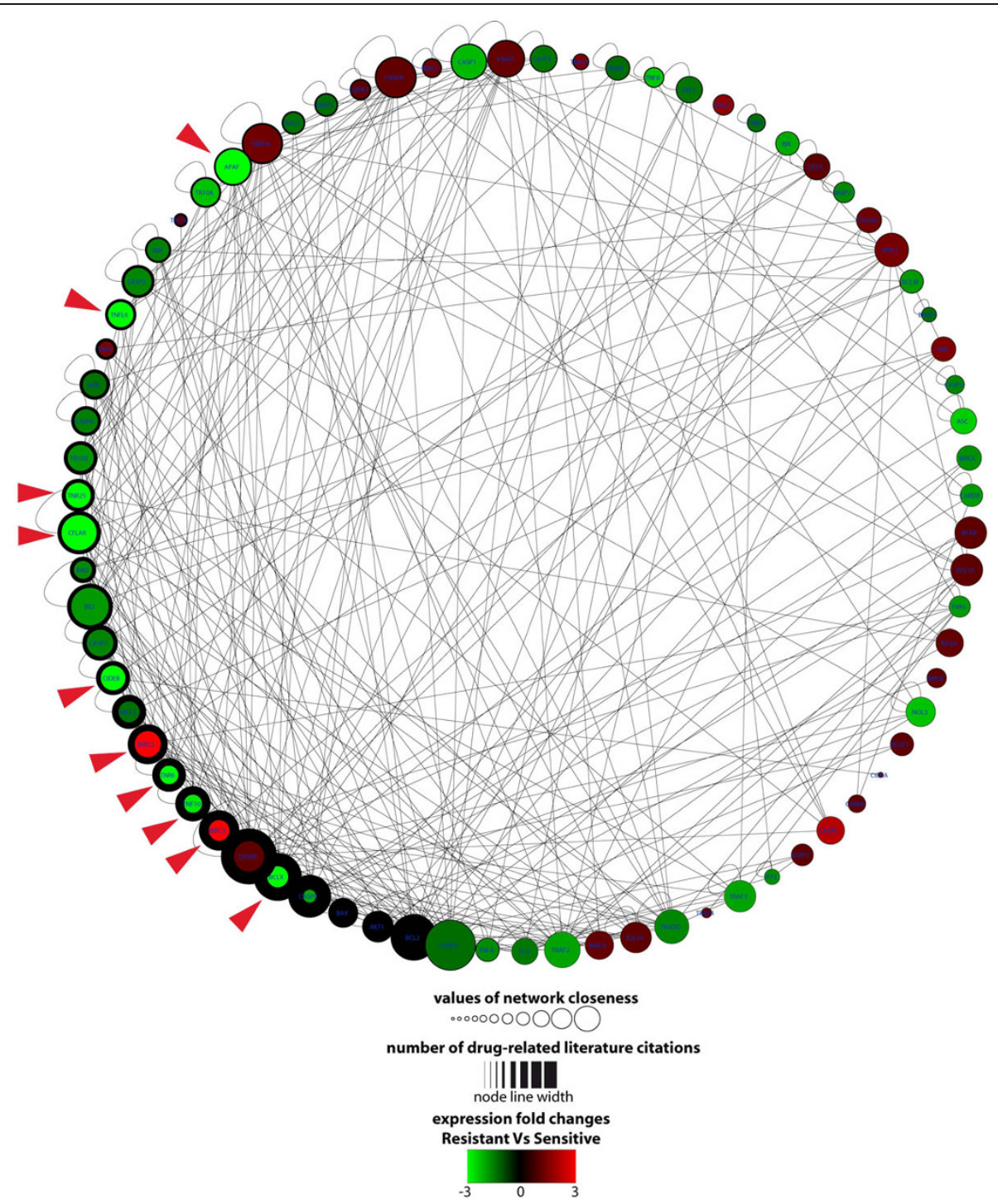

Figure 2 Correlation among network centrality, expression and involvement in drug response. yFiles Circular Layout of the AM network that emphasizes the nodes with high closeness and high involvement in drug response. The nodes with high overall drug response (number of literature citations for drugs) are localized on the left half of the circle (attribute circle layout, based on number of literature citations for drugs). The size of the nodes is related to network closeness. The colours indicate the expression fold change of AM genes (chemoresistant vs chemosensitive), according to the bar shown below the network. The node line width indicates the number of literature citations for drugs. As shown by red arrows, all nodes with altered expression are characterized by high closeness and involvement in drug response.

common to both resistant and sensitive classes and different respect to controls: many of these genes are involved in an increased response to chemotherapy (e.g., the down regulation of BAG3, BIRC4, BIRC8, BNIP family, CFLAR) [49,50]. Furthermore the downregulation of DAPK1, TNFRSF11B, TNFSF8, TP73 could be considered as a CR marker: these genes are commonly over expressed in hematopoietic malignancies, but are down regulated in patients after chemotherapy [24-26]. The detection of proapoptotic BCL10 in CR CD34+ cells, but not in normal donors (Tables 1 and 3; Figure $1)$, could be related to their proneness to death induction. Dysregulation of this gene is known to be involved in some types of haematological malignancies [51]. These observations suggest that non-leukemic CD34+ cells from CR AML patients have an expression profile apparently prone to apoptosis and to a positive response to chemotherapy. This molecular feature satisfactorily explains their positive response to in vivo chemotherapy induction, leading these patients to the first apparent CR. Plausibly, the molecular differences observed between chemoresistant and chemosensitive (acquired de novo mutations or residual presence of leukemic cells) could be responsible for the de novo 


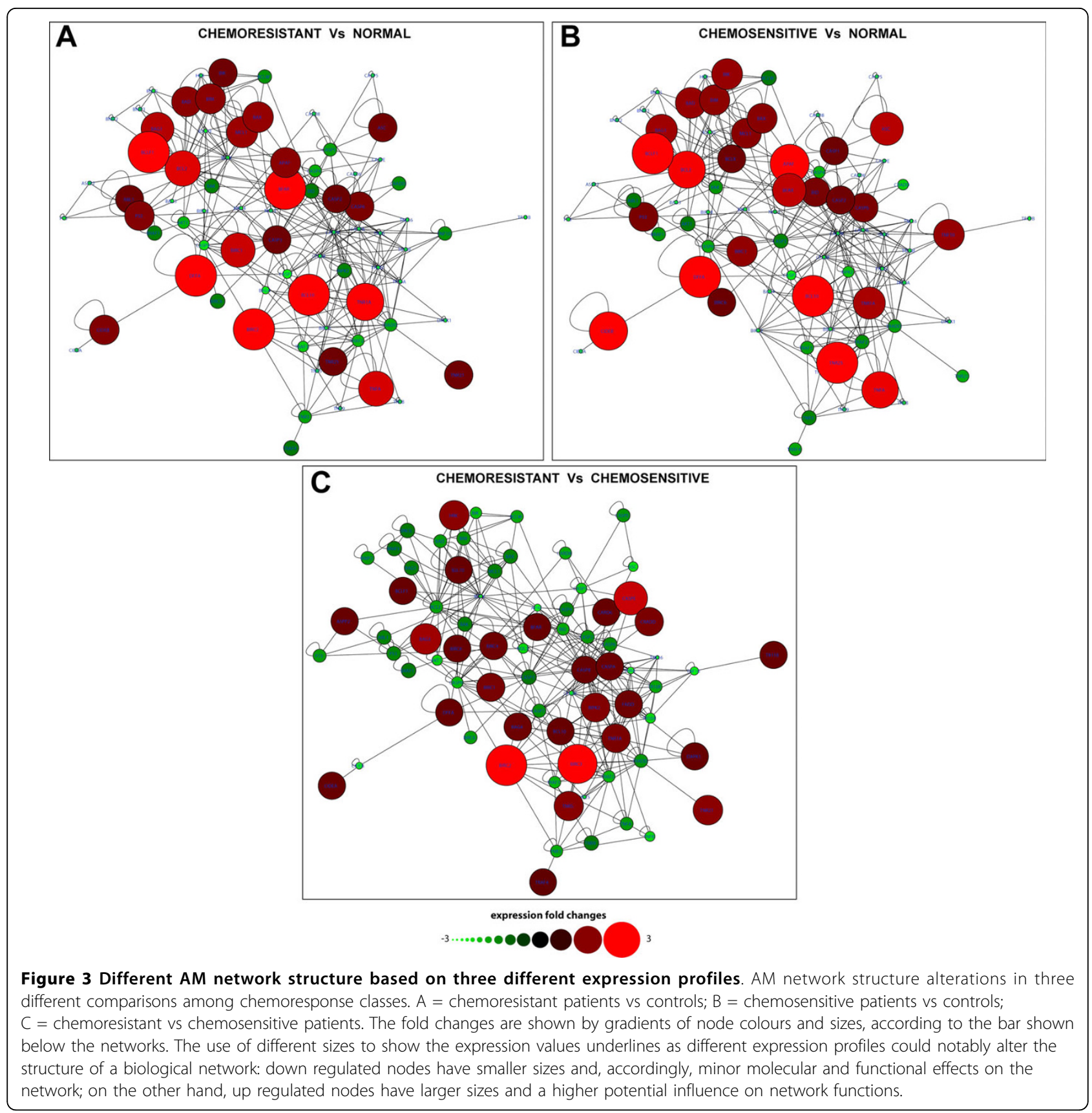

acquired resistant phenotype, and accordingly for the relapse. Unsurprisingly, these differentially expressed genes are characterized by high centrality within the AM network. Furthermore, many published data link these genes to drug response, suggesting that they perform a critical role within AM signalling activated by pharmacological treatments. Taken together these data strongly suggest that the topology of AM network is strictly related to its proper biological functioning. Malfunctioning of these central nodes affects the stability of the network and profoundly modify the physiological cell behaviour. In agreement with other published data, our results suggest that tumour-related defects in AM hubs are preferentially selected [16,52]. The functional impairment of a few nodes, which control directly or indirectly the activities of many others in the context of the co-occurrence of multiple genetic defects, could represent a selective advantage during neoplastic transformation and in response to pharmacological treatment. The differential expression of topologically important AM nodes in CR AML patients could seriously impair the physiological equilibrium of AM. 
CD34+ cells from both classes of CR AML patients showed AM network features that make these cells prone to apoptosis: accordingly, it is not surprising that these patients were in apparent CR after the first cycle of chemotherapy induction and consolidation (Figure 3). On the other hand, the AM network of the resistant class showed some critical features respect to that of the sensitive class: by impairing proapoptotic components and activating prosurvival nodes, these differences could reduce the ability of these cells to appropriately respond to death stimuli (Figure 3). Some of these AM genes are known to be dysregulated in leukaemia: up regulation of BIRC3 and down regulation of APAF1, CIDEB, FAS, TNFRSF25, TNFSF10 were previously identified by our group as specific alterations of AM genes in leukemic cells [16].

\section{Conclusions}

The ability of some cancer cell types to elude pharmacological apoptosis induction is apparently based on molecular mechanisms, similar to those involved in escaping physiological cell death. In this scenario, some AM genes represent critical nodes for the proper response to chemotherapy. Their dysregulation in HSCs from CR AML patients could be related to their high mobilizing ability, in vitro chemoresistance and high relapse rates. This molecular phenotype could result from de novo mutations, selected by the treatment, or be due to residual leukemic cells, positively selected by the cytokines used for mobilization. The AM expression profile of CD34+ cells seems to discriminate CR AML patients from normal controls, as well as in vitro chemoresistant CR AML patients from those who are sensitive. These differences affect some critical nodes of the AM network and could represent one of the causes of the differential in vitro resistance of these cells. Accordingly, AM profiling before chemotherapy and transplantation could allow the identification of patients with a genotype highly predisposing to relapse, in order to treat them by different, specifically designed, therapies. Specifically, low expression levels of APAF1, CD40LG, CIDEB, FAS, FASL, TNFRSF25, TNFSF10 and up regulation of BIRC2 and BIRC3 could pinpoint patients who are prone to relapse. Otherwise, the characterization of the genotype of the cells from $\mathrm{PB}$ could demonstrate the characteristic molecular signature of leukemic cells and accordingly guide the design of the therapeutic strategy.

\section{Additional material}

Additional file 1: Clinical characteristics of the patients.

\section{Abbreviations}

AM: Apoptotic Machinery; AML: Acute Myeloid Leukaemia; BFU-E: Burst Forming Units Erythroid; BM: Bone Marrow; CFU-E: Colony-Forming-Units Erythroid; CFU-GM: Colony Forming Unit Granulocyte-Macrophage; CR: Complete Remission; CSF: Colony-Stimulating Factor; G-CSF: Granulocyte Colony-Stimulating Factor; GM-CSF: Granulocyte-Macrophage ColonyStimulating Factor; HSC: Hematopoietic Stem Cells; MRD: Minimal Residual Disease; PB: Peripheral Blood; VLA: Very Late Antigen.

\section{Acknowledgements}

This project was financed with funds from Ministero dell'Università e della Ricerca Scientifica e Tecnologica to MP (in particular, FIRB 2007: Dalla Proteomica alla Biologia Cellulare; FAR 2007: Generation of a technological platform to study the effects of antineoplastic drugs and to investigate their potential efficacy as neuroprotective agents; PRA 2007: Caratterizzazione delle Omiche del Macchinario Apoptotico e dell'Apparato di Trascrizione: ruolo biologico dei microRNA e loro coinvolgimento in Patologia). We thank Prof S Stefani and her Collaborators (Dipartimento di Scienze

Microbiologiche, Università degli Studi di Catania) for allowing the use of their Real Time Instrument for some of our experiments. Finally, we acknowledge the technical collaboration of Mrs M Cocimano, Mr L Messina, Mr F Mondio, Mr A Vasta.

\section{Author details}

'Dipartimento di Scienze BioMediche, Unità di BioMedicina Molecolare Genomica e dei Sistemi Complessi, Genetica, Biologia Computazionale G Sichel, Via Santa Sofia 87, 95123 Catania, Italy, EU. 'Dipartimento di Scienze BioMediche, Sezione di Ematologia, 95125 Catania, Italy, EU.

\section{Authors' contributions}

MP conceived and coordinated the project with the critical collaboration of MR and GM. MP, MR, GM, CDP designed experiments, the other Researchers performed them. All Authors contributed to the critical revision of the data. $M P$ and MR wrote the paper, that was revised and approved by all Authors (MR, GA, RA, DB, MRG, LRD, AM, LS, LS, CC, MGC, CDP, GM, MP).

\section{Competing interests}

The authors declare that they have no competing interests.

Received: 12 March 2010 Accepted: 19 July 2010

Published: 19 July 2010

\section{References}

1. Mrózek K, Bloomfield CD: Chromosome aberrations, gene mutations and expression changes, and prognosis in adult acute myeloid leukemia. Hematology Am Soc Hematol Educ Program 2006, 169-77.

2. Mrózek K: Cytogenetic, molecular genetic, and clinical characteristics of acute myeloid leukemia with a complex karyotype. Semin Oncol 2008, 35(4):365-77.

3. Chauncey TR: Drug resistance mechanisms in acute leukemia. Curr Opin Oncol 2001, 13(1):21-6.

4. Marie JP: Drug resistance in hematologic malignancies. Curr Opin Oncol 2001, 13(6):463-9.

5. Estey E, Döhner H: Acute myeloid leukaemia. Lancet 2006, 368(9550): 1894-907.

6. Jabbour EJ, Estey E, Kantarjian HM: Adult acute myeloid leukemia. Mayo Clin Proc 2006, 81(2):247-60.

7. Meads MB, Hazlehurst LA, Dalton WS: The bone marrow microenvironment as a tumor sanctuary and contributor to drug resistance. Clin Cancer Res 2008, 14(9):2519-26.

8. Li ZW, Dalton WS: Tumor microenvironment and drug resistance in hematologic malignancies. Blood Rev 2006, 20(6):333-42.

9. Papayannopoulou T, Scadden DT: Stem-cell ecology and stem cells in motion. Blood 2008, 111(8):3923-30

10. Gazitt Y: Homing and mobilization of hematopoietic stem cells and hematopoietic cancer cells are mirror image processes, utilizing similar signaling pathways and occurring concurrently: circulating cancer cells constitute an ideal target for concurrent treatment with chemotherapy and antilineage-specific antibodies. Leukemia 2004, 18(1):1-10. 
11. Keating $S$, Suciu $S$, de Witte $T$, Zittoun R, Mandelli F, Belhabri A, Amadori $S$, Fibbe W, Gallo E, Fillet G, Varet B, Meloni G, Hagemeijer A, Fazi P, Solbu G, Willemze R, EORTC Leukemia Group, GIMEMA Leukemia Group: The stem cell mobilizing capacity of patients with acute myeloid leukemia in complete remission correlates with relapse risk: results of the EORTC GIMEMA AML-10 trial. Leukemia 2003, 17:60-67.

12. Feller N, Schuurhuis GJ, van der Pol MA, Westra G, Weijers GW, van Stijn A Huijgens PC, Ossenkoppele GJ: High percentage of CD34-positive cells in autologous AML peripheral blood stem cell products reflects inadequate in vivo purging and low chemotherapeutic toxicity in a subgroup of patients with poor clinical outcome. Leukemia 2003, 17:68-75.

13. Grimwade D, Walker H, Oliver F, Wheatley K, Harrison C, Harrison G, Rees J, Hann I, Stevens R, Burnett A, Goldstone A: The Importance of Diagnostic Cytogenetics on Outcome in AML: Analysis of 1,612 Patients Entered Into the MRC AML 10 Trial. Blood 1998, 92:2322-2333.

14. Rodriguez-Nieto S, Zhivotovsky B: Role of Alterations in the Apoptotic Machinery in Sensitivity of Cancer Cells to Treatment. Current Pharmaceutical Design 2006, 12:4411-4425.

15. Mellor HR, Callaghan R: Resistance to Chemotherapy in Cancer: A Complex and Integrated Cellular Response. Pharmacology 2008, 81:275-300.

16. Di Pietro C, Ragusa M, Barbagallo D, Duro LR, Guglielmino MR, Majorana A, Angelica R, Scalia M, Statello L, Salito L, Tomasello L, Pernagallo S, Valenti S, D’Agostino V, Triberio P, Tandurella I, Palumbo GA, La Cava P, Cafiso V, Bertuccio T, Santagati M, Li Destri G, Lanzafame S, Di Raimondo F, Stefani S, Mishra B, Purrello M: The apoptotic machinery as a biological complex system: analysis of its omics and evolution, identification of candidate genes for fourteen major types of cancer, and experimental validation in CML and neuroblastoma. BMC Med Genomics 2009, 2(1):20.

17. Livak KJ, Schmittgen TD: Analysis of relative gene expression data using real-time quantitative PCR and the 2(-Delta Delta C(T)) Method. Methods 2001, 25(4):402-8.

18. Saeed Al, Sharov V, White J, Li J, Liang W, Bhagabati N, Braisted J, Klapa M, Currier T, Thiagarajan M, Sturn A, Snuffin M, Rezantsev A, Popov D, Ryltsov A, Kostukovich E, Borisovsky I, Liu Z, Vinsavich A, Trush V, Quackenbush J: TM4: a free, open-source system for microarray data management and analysis. Biotechniques 2003, 34(2):374-8.

19. Shannon P, Markiel A, Ozier O, Baliga NS, Wang JT, Ramage D, Amin N, Schwikowski B, Ideker T: Cytoscape: a software environment for integrated models of biomolecular interaction networks. Genome Res 2003, 13:2498-2504.

20. Hernandez-Toro J, Prieto C, De las Rivas J: APID2NET: unified interactome graphic analyzer. Bioinformatics 2007, 23(18):2495-7.

21. Assenov Y, Ramírez F, Schelhorn SE, Lengauer T, Albrecht M: Computing topological parameters of biological networks. Bioinformatics 2008, 24(2):282-284

22. Brandes U, Erlebach T: Network Analysis Berlin: Springer Verlag 2005.

23. Mattingly CJ, Rosenstein MC, Davis AP, Colby GT, Forrest JN, Boyer JL: The Comparative Toxicogenomics Database: a cross-species resource for building chemical-gene interaction networks. Toxicol Sci 2006, 92(2):587-95

24. Nagy $B$, Lundán $T$, Larramendy $M L$, Aalto $Y$, Zhu $Y$, Niini $T$, Edgren $H$, Ferrer A, Vilpo J, Elonen E, Vettenranta K, Franssila K, Knuutila S: Abnormal expression of apoptosis-related genes in haematological malignancies: overexpression of MYC is poor prognostic sign in mantle cell lymphoma. Br J Haematol 2003, 120(3):434-41.

25. Gattei $V$, Degan M, Gloghini A, De luliis A, Improta S, Rossi FM, Aldinucci D, Perin V, Serraino D, Babare R, Zagonel V, Gruss HJ, Carbone A, Pinto A: CD30 ligand is frequently expressed in human hematopoietic malignancies of myeloid and lymphoid origin. Blood 1997, 89(6):2048-59.

26. Lucena-Araujo AR, Panepucci RA, dos Santos GA, Jácomo RH, SantanaLemos BA, Lima AS, Garcia AB, Araújo AG, Falcão RP, Rego EM: The expression of DeltaNTP73, TATP73 and TP53 genes in acute myeloid leukaemia is associated with recurrent cytogenetic abnormalities and in vitro susceptibility to cytarabine cytotoxicity. $\mathrm{Br} J$ Haematol 2008 , 142(1):74-8.

27. Bonelli P, Petrella A, Rosati A, Romano MF, Lerose R, Pagliuca MG, Amelio T, Festa M, Martire G, Venuta S, Turco MC, Leone A: BAG3 protein regulates stress-induced apoptosis in normal and neoplastic leukocytes. Leukemia 2004, 18(2):358-60.
28. Mercier I, Vuolo M, Madan R, Xue X, Levalley AJ, Ashton AW, Jasmin JF, Czaja MT, Lin EY, Armstrong RC, Pollard JW, Kitsis RN: ARC, an apoptosis suppressor limited to terminally differentiated cells, is induced in human breast cancer and confers chemo- and radiation-resistance. Cell Death Differ 2005, 12(6):682-6.

29. Cho D, Kim YI, Kang JS, Hahm E, Yang Y, Kim D, Kim S, Kim YS, Hur D, Park H, Hwang Yl, Kim TS, Lee WJ: Resistance to cytotoxic chemotherapy is induced by NK cells in non-Hodgkin's Lymphoma Cells. J Clin Immunol 2004, 24(5):553-60.

30. Vaziri SA, Grabowski DR, Tabata M, Holmes KA, Sterk J, Takigawa N, Bukowski RM, Ganapathi MK, Ganapathi R: C-IAP1 is overexpressed in HL60 cells selected for doxorubicin resistance: effects on etoposideinduced apoptosis. Anticancer Res 2003, 23(5A):3657-61.

31. Valdez BC, Murray D, Ramdas L, de Lima M, Jones R, Kornblau S, Betancourt D, Li Y, Champlin RE, Andersson BS: Altered gene expression in busulfan-resistant human myeloid leukemia. Leuk Res 2008, 32(11):1684-97.

32. Corvaro M, Fuoco C, Wagner M, Cecconi F: Analysis of apoptosome dysregulation in pancreatic cancer and of its role in chemoresistance. Cancer Biol Ther 2007, 6(2):209-17.

33. Longley DB, Wilson TR, McEwan M, Allen WL, McDermott U, Galligan L, Johnston PG: c-FLIP inhibits chemotherapy-induced colorectal cancer cell death. Oncogene 2006, 25(6):838-48.

34. Nadal C, Maurel J, Gallego R, Castells A, Longarón R, Marmol M, Sanz S, Molina R, Martin-Richard M, Gascón P: FAS/FAS ligand ratio: a marker of oxaliplatin-based intrinsic and acquired resistance in advanced colorectal cancer. Clin Cancer Res 2005, 11(13):4770-4.

35. Joy MP, Brock A, Ingber DE, Huang S: High-betweenness proteins in the yeast protein interaction network. J Biomed Biotechnol 2005, 2005(2):96-103.

36. Luqmani YA: Mechanisms of drug resistance in cancer chemotherapy. Med Princ Pract 2005, 14(Suppl 1):35-48.

37. Meads MB, Gatenby RA, Dalton WS: Environment-mediated drug resistance: a major contributor to minimal residual disease. Nat Rev Cancer 2009, 9: 665-74.

38. Kopp HG, Avecilla ST, Hooper AT, Rafii S: The bone marrow vascular niche: home of HSC differentiation and mobilization. Physiology 2005, 20:349-56.

39. Bociek RG, Armitage JO: Hematopoietic growth factors. CA Cancer J Clin 1996, 46(3):165-84

40. Lotem J, Sachs L: Cytokine control of developmental programs in normal hematopoiesis and leukemia. Oncogene 2002, 21(21):3284-94.

41. Liongue C, Wright C, Russell AP, Ward AC: Granulocyte colony-stimulating factor receptor: stimulating granulopoiesis and much more. Int J Biochem Cell Biol 2009, 41(12):2372-5.

42. Derouet M, Thomas L, Cross A, Moots RJ, Edwards SW: Granulocyte macrophage colony-stimulating factor signaling and proteasome inhibition delay neutrophil apoptosis by increasing the stability of Mcl-1. J Biol Chem 2004, 279(26):26915-21.

43. Kotone-Miyahara Y, Yamashita K, Lee KK, Yonehara S, Uchiyama T, Sasada M, Takahashi A: Short-term delay of Fas-stimulated apoptosis by GM-CSF as a result of temporary suppression of FADD recruitment in neutrophils: evidence implicating phosphatidylinositol 3-kinase and MEK1-ERK1/2 pathways downstream of classical protein kinase C. J Leukoc Biol 2004, 76(5):1047-56.

44. Gu L, Chiang KY, Zhu N, Findley HW, Zhou M: Contribution of STAT3 to the activation of survivin by GM-CSF in CD34+ cell lines. Exp Hematol 2007, 35(6):957-66.

45. Sachs L, Lotem J: Control of Programmed Cell Death in Normal and Leukemic Cells: New Implications for Therapy. Blood 1993, , 82: 15-21.

46. Eisele L, Klein-Hitpass L, Chatzimanolis N, Opalka B, Boes T, Seeber S, Moritz T, Flasshove M: Differential expression of drug-resistance-related genes between sensitive and resistant blasts in acute myeloid leukemia. Acta Haematol 2007, 117(1):8-15.

47. Murayama T, Imoto S, Natazuka T, Chihara K, Matsui T: Proliferative reaction of myelogenous leukemia cells with cytokines G-CSF, GM-CSF, M-CSF, SCF and TPO. Leuk Res 1998, 22(6):557-60.

48. Inukai T, Sugita K, lijima K, Goi K, Tezuka T, Kojika S, Kagami K, Mori T, Kinoshita A, Suzuki T, O-Koyama T, Nakazawa S: Leukemic cells with 11q23 translocations express granulocyte colony-stimulating factor (G-CSF) receptor and their proliferation is stimulated with G-CSF. Leukemia 1998, 12(3):382-9. 
49. Seal S, Hockenbery DM, Spaulding EY, Kiem HP, Abbassi N, Deeg HJ: Differential responses of FLIPLong and FLIPShort-overexpressing human myeloid leukemia cells to TNF-alpha and TRAIL-initiated apoptotic signals. Exp Hematol 2008, 36(12):1660-72.

50. Zang F, Sun B, Zhao X, Niu R, Zhang S, Yu M, Wei X, Zhang L: Critical role for c-FLIP(L) on Fas resistance in colon carcinoma cell line HT-29. Cell Biol Int 2008, 32(3):329-36.

51. Grimwade D, Du MQ, Langabeer S, Rogers J, Solomon E: Screening for mutations of Bcl10 in leukaemia. Br J Haematol 2000, 109(3):611-5.

52. Cui $Q$, Ma Y, Jaramillo M, Bari H, Awan A, Yang S, Zhang S, Liu L, Lu M, O'Connor-McCourt M, Purisima EO, Wang E: A map of human cancer signaling. Mol Syst Biol 2007, 3:152.

\section{Pre-publication history}

The pre-publication history for this paper can be accessed here: http://www.biomedcentral.com/1471-2407/10/377/prepub

doi:10.1186/1471-2407-10-377

Cite this article as: Ragusa et al:: Expression profile and specific network features of the apoptotic machinery explain relapse of acute myeloid leukemia after chemotherapy. BMC Cancer 2010 10:377.

\section{Submit your next manuscript to BioMed Central} and take full advantage of:

- Convenient online submission

- Thorough peer review

- No space constraints or color figure charges

- Immediate publication on acceptance

- Inclusion in PubMed, CAS, Scopus and Google Scholar

- Research which is freely available for redistribution

Submit your manuscript at www.biomedcentral.com/submit 